\title{
Rapamycin inhibits spermatogenesis by changing the autophagy status through suppressing mechanistic target of rapamycin-p70S6 kinase in male rats
}

\author{
SHANGJING LIU ${ }^{1 *}$, LONGXIAN HUANG $^{2 *}$, YANQING GENG ${ }^{1}$, JUNLIN HE ${ }^{1}$, XUEMEI CHEN ${ }^{1}$, \\ HAO XU ${ }^{1}$, RONG $\mathrm{LI}^{3}$, YINGXIONG WANG ${ }^{1}$, YUBIN DING ${ }^{1}$ and XUEQING LIU ${ }^{1}$ \\ ${ }^{1}$ Laboratory of Reproductive Biology, School of Public Health and Management; ${ }^{2}$ Department of Respiration, \\ The First Branch of The First Affiliated Hospital, Chongqing Medical University; ${ }^{3}$ Department of Obstetrics and \\ Gynecology, The First Affiliated Hospital of Chongqing Medical University, Chongqing 400016, P.R. China
}

Received September 11, 2016; Accepted May 24, 2017

DOI: $10.3892 / \mathrm{mmr} .2017 .7120$

\begin{abstract}
Rapamycin (sirolimus) is an antiproliferative drug that has been widely used in the clinic as an immunosuppressant and a potential anticancer agent. Certain reports have indicated that rapamycin may induce male infertility through impairing sperm quality. The present study investigated the mechanism of male infertility caused by rapamycin and examined whether withdrawal of rapamycin could recover the number of sperm in rats. Male Sprague-Dawley rats $(n=100)$ were divided randomly into 5 groups: 3 rapamycin-treated groups $(2,4$ and $6 \mathrm{mg} / \mathrm{kg})$ and 2 control groups [Blank and dimethyl sulfoxide (DMSO)]. Organ coefficients of the testes, number of sperm and hematoxylin-eosin staining analyses demonstrated that rapamycin treatment markedly damaged the structure of the seminiferous tubule and reduced the number of sperm. Immunohistochemistry of mechanistic target of rapamycin (mTOR) and Ki67 in testes tissue, and western blotting of phosphorylated-p70S6K and p70S6K, supported the hypothesis that rapamycin causes sperm reduction through inhibiting proliferation of spermatogonia. Unfortunately, 24 weeks after cessation of rapamycin treatment, only the number of sperm in $2 \mathrm{mg} / \mathrm{kg}$ group was restored back to the normal level. In addition, to the best of our knowledge, the present study was the first to demonstrate that low doses rapamycin leads to activation of autophagy in rat testes. This may be a self-protective mechanism of the cell in response to external stress. Thus, spermatogenesis can be recovered in the
\end{abstract}

Correspondence to: Professor Xueqing Liu, Laboratory of Reproductive Biology, School of Public Health and Management, Chongqing Medical University, 1 Yixueyuan Road, Yuzhong, Chongqing 400016, P.R. China

E-mail: cqmu_liuxueqing@126.com

*Contributed equally

Key words: rapamycin (sirolimus), mechanistic target of rapamycin, sperm, spermatogenesis, autophagy testes from rats in the low dose group. High doses of rapamycin resulted in excessive consumption of autophagy proteins, and the damage could not be compensated. In addition, it was revealed that cell apoptosis increased after treatment with rapamycin. In conclusion, the present study demonstrated that rapamycin inhibits spermatogenesis through suppressing phosphorylation of p70S6K and changing the autophagy status, ultimately reducing the number of sperm. These findings provide important guidance for the clinical application of rapamycin.

\section{Introduction}

Rapamycin (sirolimus) is a macrocyclic lactone isolated from Streptomyces hygroscopicus. It has been widely used in the clinic as an antiproliferative drug, an immunosuppressant and a potential anticancer agent $(1,2)$. Recent reports have indicated that rapamycin may alter sex hormone levels and impair sperm quality (3). Rovira et al (4) demonstrated that $1 \mathrm{mg} / \mathrm{kg}$ rapamycin could induce gonadal dysfunction, seminiferous tubule dystrophy and reversible spermatogenesis blockade in rats. Deutsch et al (5) reported a case of sirolimus-associated infertility and suggested that sirolimus may negatively influence spermatogenesis. However, the mechanism is unclear. However, it is not known whether damage of the testes and the reversibility of spermatogenesis blockade are dose-dependent. Thus, further research on the underlying mechanism and potential dose-dependent effect is required.

Spermatogenesis is a dynamic and multistep process of germ cell proliferation and differentiation by which spermatozoa are produced from primordial germ cells (6). This process is regulated by some key regulators such as c-Kit, azoospermia factors and deleted in azoospermia associated protein 1 (7-9). Mechanistic target of rapamycin (mTOR) is a central regulator of cell growth which is involved in growth-associated processes including translation, transcription and autophagy $(2,10)$. Rapamycin is a specific inhibitor of mTOR, which can specifically bind to mTOR and downregulate its function (11).

The present study focused on the potential mechanism of the spermatogenesis blockade, and demonstrated that rapamycin inhibits the proliferation of spermatogonia through 
suppressing the mTOR-p70S6 Kinase (p70S6K) signaling pathway in male rats. Furthermore, the dose-dependent effect of rapamycin on spermatogenesis dysfunction was investigated; the results revealed that impairment of the testes could be partially recovered after withdrawal of rapamycin treatment at a low dose $(2 \mathrm{mg} / \mathrm{kg})$.

\section{Materials and methods}

Animal model and rapamycin administration. The rats used in this study were obtained from the Experimental Animal Center of Chongqing Medical University (Chongqing, China; certificate no. SCXK (YU) 20070001). All rats used had free access to food and water, and housed under the specific pathogen-free condition (12-h light/dark cycle with humidity of $55 \pm 5 \%$ at $25 \pm 2^{\circ} \mathrm{C}$ ). The animal procedures were carried out in accordance with and approved by the Ethics Committee of Chongqing Medical University.

Sexually mature male Sprague-Dawley rats (weight, $200 \mathrm{~g}$; age, 8 weeks; $n=100$ ) were used in this study. During the experimental period, the animals were randomly assigned to 5 groups: 2 control groups [Blank, dimethyl sulfoxide (DMSO)] and 3 rapamycin-treated groups $(2,4$ and $6 \mathrm{mg} / \mathrm{kg})$. Sodium rapamycin (>99\% pure) was obtained from Taizhou Crene Biotechnology Co., Ltd. (Shanghai, China), dissolved in $0.9 \% \mathrm{NaCl}$ solution containing $1 \%$ DMSO at a concentration of $1 \mathrm{mg} / \mathrm{ml}$ and stored at $-20^{\circ} \mathrm{C}$. The dosage, duration and administration of rapamycin were based on previous reports in rats $(3,12-14)$. To investigate the dose-dependent effect of rapamycin, daily intraperitoneal injections (i.p.) at dosages of 2, 4 and $6 \mathrm{mg} / \mathrm{kg}$ were performed for 4 weeks. As controls, the DMSO group received $1 \mathrm{ml}$ vehicle solution per day, and the Blank group received no treatment.

At the end of the rapamycin exposure period (4 weeks), 10 rats in each group were sacrificed for morphological, histological and molecular biological examinations of the testes, and the other 10 rats in each group were assigned to withdraw rapamycin for 24 weeks for further observation. A detailed experimental protocol is presented in Table I.

Organ coefficients of testes. After treatment with rapamycin or DMSO for 4 weeks, 10 rats from each group were weighed separately using an electronic balance. Following anesthesia with $1 \mathrm{ml} \mathrm{10 \%}$ chloral hydrate (Biomart, Zhejiang, China), the testes were removed and weighed according to a previous report (15). Organ coefficients of testes were calculated through this formula: Organ coefficients=organ weight/body weight $\mathrm{x} 100$ (16).

Number of sperm. The left cauda epididymis of rats was dissected and then sectioned into small slices to release the spermatozoa into $1 \mathrm{ml} 0.9 \% \mathrm{NaCl}$ solution. Sperm suspension (5 $\mu \mathrm{l})$ was diluted with $1 \mathrm{ml} 0.9 \% \mathrm{NaCl}$ solution and incubated at $37^{\circ} \mathrm{C}$ for $1 \mathrm{~h}$. The diluted sperm suspension was transferred into Neubauer hemocytometer's chamber and sperm heads were counted under an inverted microscope (Olympus IX51) according to World Health Organisation guidelines (17). Data were recorded as the number of sperm per $\mathrm{ml}$.

Histological examination. The testes were removed and fixed in $4 \%$ formaldehyde solution at room temperature. Tissue samples were dehydrated and embedded in paraffin according to standard histological procedures (18). Serial $5-\mu \mathrm{m}$ cross-sections were prepared from each testis. The sections were mounted and stained with Hematoxylin for $1 \mathrm{~min}$ and Eosin for $30 \mathrm{sec}$ (HE staining) at room temperature. Images were captured under an Olympus BX40 microscope.

Immunohistochemistry. The tissue sections were deparaffinized in xylene and rehydrated in descending concentrations of ethanol, followed by antigen retrieval and cooling at room temperature. Endogenous peroxidase was inhibited by incubation with $3 \%$ hydrogen peroxide for $10 \mathrm{~min}$ at room temperature. The tissue sections were blocked in $10 \%$ normal goat serum for $30 \mathrm{~min}$ and incubated with a primary antibody at $4^{\circ} \mathrm{C}$ overnight. The primary antibodies used in the present study were a rabbit monoclonal anti-mTOR antibody at a dilution of 1:80 (cat. no. \#2983; Cell Signaling Technology, Inc., Danvers, MA, USA) and a rabbit monoclonal anti-Ki67 antibody at a dilution of 1:50 (cat. no. ab16667; Abcam, Cambridge, USA). The following process was performed using a Histostain ${ }^{\mathrm{TM}}$-Plus kit (SP9001; OriGene Technologies, Inc., Rockville, MD, USA) and chromogenic reaction was conducted with 3,30-diaminobenzidine (ZLI-9018; ZSGB-BIO, China). After that, the slices were counterstained with HE, dehydrated after hydrochloric alcohol differentiation and mounted with resinene. Tissue sections were imaged under an Olympus BX40 microscope.

Protein extraction and western blotting. Proteins were extracted from the testes of rats using radioimmunoprecipitation assay reagent (P0013B; Beyotime Institute of Biotechnology, Haimen, China) containing 1\% PMSF (ST506, Beyotime Institute of Biotechnology) to prevent the degradation of protein. Briefly, tissue homogenates were centrifuged at $10,000 \mathrm{x} \mathrm{g}$ and $4^{\circ} \mathrm{C}$ for $15 \mathrm{~min}$, then the supernatant was collected. Protein concentrations were measured by the Bicinchoninic Acid method (Beyotime Institute of Biotechnology). Protein samples (50 $\mu \mathrm{g})$ were mixed with 5X SDS-PAGE Sample Loading Buffer and boiled for $5 \mathrm{~min}$ before being loaded onto a 10\% SDS-PAGE gel. After electrophoresis, proteins were transferred to polyvinylidene fluoride membrane (EMD Millipore, Billerica, MA, USA). Membranes were cut according to the marker and incubated in 5\% nonfat milk with PBS with Tween-20 (PBST) for $1 \mathrm{~h}$ at room temperature with agitation to block nonspecific protein binding. The following rabbit monoclonal primary antibodies were used: p70S6K at a dilution of 1:1,000 (cat. no. \#9202; Cell Signaling Technology, Inc.), phosphorylated (p)-p70S6K at a dilution of 1:1,000 (cat. no. \#9234; Cell Signaling Technology, Inc.), microtubule-associated protein 1A/1B-light chain 3 (LC3) at a dilution of 1:1,000 (cat. no. \#3868S; Cell Signal Technology, Inc.), P62 at a dilution of 1:500 (cat. no. \#5114S; Cell Signal Technology, Inc.) and Bcl-2 X associated protein (Bax) at a dilution of 1:1,000 (cat. no. \#04-434; EMD Millipore). Additional primary antibodies included B-cell lymphoma 2 (Bcl-2) rabbit polyclonal at a dilution of 1:600 (cat. no. AB20521a; Sangon Biotech, China) and $\beta$-actin mouse monoclonal at a dilution of 1:2,000 (cat. no. A5441; Sigma-Aldrich; Merck KGaA, Darmstadt, Germany; internal loading control). Membranes were incubated with primary antibodies overnight at $4^{\circ} \mathrm{C}$ and then washed in PBST for $3 \times 10$ min prior to incubation with the corresponding secondary antibody (diluted in 5\% non-fat 
Table I. Experimental protocol.

\begin{tabular}{|c|c|c|c|}
\hline Group & $\mathrm{N}$ & $\begin{array}{l}\text { Weight of rats prior } \\
\text { to treatment }(\mathrm{g})\end{array}$ & Treatment \\
\hline Blank & 20 & $197.53 \pm 7.45$ & $\begin{array}{l}10 \text { rats: no treatment for } 4 \text { weeks } \\
10 \text { rats: no treatment for } 4 \text { weeks }+24 \text { weeks }\end{array}$ \\
\hline DMSO & 20 & $200.53 \pm 10.85$ & $\begin{array}{l}10 \text { rats: DMSO i.p. for } 4 \text { weeks } \\
10 \text { rats: DMSO i.p. for } 4 \text { weeks + withdrawal for } 24 \text { weeks }\end{array}$ \\
\hline $2 \mathrm{mg} / \mathrm{kg}$ & 20 & $203.47 \pm 9.56$ & $\begin{array}{l}10 \text { rats: } 2 \mathrm{mg} / \mathrm{kg} / \text { day rapamycin i.p. for } 4 \text { weeks } \\
10 \text { rats: } 2 \mathrm{mg} / \mathrm{kg} / \text { day rapamycin i.p. for } 4 \text { weeks + withdrawal for } 24 \text { weeks }\end{array}$ \\
\hline $4 \mathrm{mg} / \mathrm{kg}$ & 20 & $201.70 \pm 14.29$ & $\begin{array}{l}10 \text { rats: } 4 \mathrm{mg} / \mathrm{kg} / \text { day rapamycin i.p. for } 4 \text { weeks } \\
10 \text { rats: } 4 \mathrm{mg} / \mathrm{kg} / \text { day rapamycin i.p. for } 4 \text { weeks + withdrawal for } 24 \text { weeks }\end{array}$ \\
\hline $6 \mathrm{mg} / \mathrm{kg}$ & 20 & $200.24 \pm 11.45$ & $\begin{array}{l}10 \text { rats: } 6 \mathrm{mg} / \mathrm{kg} / \text { day rapamycin i.p. for } 4 \text { weeks } \\
10 \text { rats: } 6 \mathrm{mg} / \mathrm{kg} / \text { day rapamycin i.p. for } 4 \text { weeks + withdrawal for } 24 \text { weeks }\end{array}$ \\
\hline
\end{tabular}

i.p., intraperitoneally; DMSO, dimethyl sulfoxide.

Table II. Organ coefficients of testes following the treatment with rapamycin.

\begin{tabular}{lclll}
\hline Group & $\mathrm{N}$ & \multicolumn{1}{c}{$\begin{array}{c}\text { Body weight } \\
(\mathrm{g})\end{array}$} & $\begin{array}{c}\text { Testes } \\
\text { weight }(\mathrm{g})\end{array}$ & $\begin{array}{c}\text { Organ } \\
\text { coefficients }\end{array}$ \\
\hline Blank & 10 & $249.04 \pm 8.75$ & $3.24 \pm 0.20$ & $1.30 \pm 0.07$ \\
DMSO & 10 & $250.54 \pm 12.40$ & $3.22 \pm 0.24$ & $1.28 \pm 0.09$ \\
$2 \mathrm{mg} / \mathrm{kg}$ & 10 & $255.10 \pm 8.49$ & $2.26 \pm 0.17^{\mathrm{a}}$ & $0.89 \pm 0.08^{\mathrm{a}}$ \\
$4 \mathrm{mg} / \mathrm{kg}$ & 10 & $254.76 \pm 13.35$ & $2.10 \pm 0.10^{\mathrm{a}}$ & $0.83 \pm 0.04^{\mathrm{a}}$ \\
$6 \mathrm{mg} / \mathrm{kg}$ & 10 & $256.24 \pm 11.43$ & $1.92 \pm 0.10^{\mathrm{a}}$ & $0.75 \pm 0.05^{\mathrm{a}}$ \\
\hline
\end{tabular}

Data are expressed as the mean \pm standard deviation. ${ }^{\mathrm{a}} \mathrm{P}<0.05$ vs. Blank group. DMSO, dimethyl sulfoxide.

milk) for $1 \mathrm{~h}$ at room temperature [horseradish peroxidase (HRP)-labeled goat anti-rabbit or HRP-labeled anti-mouse (cat. nos. ZDR-5306 or ZDR-5307; ZSGB-BIO; OriGene Technologies, Inc., Beijing, China)]. The positive bands were detected by chemiluminescent reaction (EMD Millipore). Image collection and densitometry analysis were performed by Quantity One version 4.6.2 analysis software (Bio-Rad Laboratories, Inc., Hercules, CA, USA).

Statistical analysis. To determine the toxicity of rapamycin on male fertility, SPSS 13.0 software (SPSS, Inc., Chicago, IL, USA) was used to conduct statistical analyses. Comparisons between groups were analyzed by one-way analysis of variance and the least significant difference post hoc test. Data are expressed as the mean \pm standard deviation. $\mathrm{P}<0.05$ was considered to indicate a statistically significant difference.

\section{Results}

Effects of rapamycin on organ coefficients of testes. After treatment with rapamycin or DMSO for 4 weeks, body weight and the testes of rats from each of the 5 groups were examined. As presented in Table II, no significant difference in body weight was found between the 5 groups. However, testes weight and organ coefficients of testes reduced remarkably in rapamycin-treated groups compared with the control groups $(\mathrm{P}<0.05)$. No statistical difference in testes weight and organ coefficients were identified between the 2,4 and $6 \mathrm{mg} / \mathrm{kg}$ groups $(\mathrm{P}>0.05)$.

Organ coefficients of testes after withdrawal of rapamycin. After the treatment of rapamycin was withdrawn for 24 weeks, body and testes weights were examined in each group. As presented in Table III, there was no significant difference in body weight between the 5 groups. Testes weight and organ coefficients of the testes were lower in the 4 and $6 \mathrm{mg} / \mathrm{kg}$ groups compared with the Blank, DMSO and $2 \mathrm{mg} / \mathrm{kg}$ groups $(\mathrm{P}<0.05)$. No significant difference in the organ coefficients of testes was identified between the Blank, DMSO and $2 \mathrm{mg} / \mathrm{kg}$ group $(\mathrm{P}>0.05)$.

Number of sperm after the treatment and withdrawal of rapamycin. To explore whether rapamycin had a negative impact on spermatogenesis, the sperm numbers of rats in each group were counted. As presented in Fig. 1, the number of sperm in rapamycin-treated groups reduced markedly compared with the Blank and DMSO groups $(\mathrm{P}<0.05)$. No difference was identified between the Blank and DMSO groups $(\mathrm{P}>0.05)$. In addition, after withdrawal of rapamycin for 24 weeks, the number of sperm in the $2 \mathrm{mg} / \mathrm{kg}$ group restored to normal level $(\mathrm{P}<0.05)$. However, sperm counts in the 4 and $6 \mathrm{mg} / \mathrm{kg}$ groups did not fully recover $(\mathrm{P}>0.05)$. In the Blank and DMSO groups, the treatment and withdrawal status did not affect the number of sperm.

Changes in histology of the testes after the treatment and withdrawal of rapamycin. As the sperm number of rapamycin-treated groups reduced remarkably, routine $\mathrm{HE}$ staining of the testes in each group was performed to determine changes in the histological structure of seminiferous tubule. Diameters of seminiferous tubules in rapamycin-treated groups was markedly smaller than the Blank and DMSO groups (Fig. 2A). In the testicular seminiferous tubules of rats 
Table III. Organ coefficients of testes following the withdrawal of rapamycin.

\begin{tabular}{lcccc}
\hline Group & $\mathrm{N}$ & $\begin{array}{c}\text { Body weight } \\
(\mathrm{g})\end{array}$ & $\begin{array}{c}\text { Testes } \\
\text { weight }(\mathrm{g})\end{array}$ & $\begin{array}{c}\text { Organ } \\
\text { coefficients }\end{array}$ \\
\hline Blank & 10 & $309.74 \pm 10.54$ & $3.50 \pm 0.18$ & $1.13 \pm 0.09$ \\
DMSO & 10 & $309.62 \pm 20.31$ & $3.51 \pm 0.13$ & $1.14 \pm 0.10$ \\
$2 \mathrm{mg} / \mathrm{kg}$ & 10 & $319.86 \pm 19.45$ & $3.36 \pm 0.09$ & $1.05 \pm 0.07$ \\
$4 \mathrm{mg} / \mathrm{kg}$ & 10 & $314.16 \pm 20.56$ & $2.35 \pm 0.14^{\mathrm{a}}$ & $0.73 \pm 0.04^{\mathrm{a}}$ \\
$6 \mathrm{mg} / \mathrm{kg}$ & 10 & $314.68 \pm 17.94$ & $2.10 \pm 0.11^{\mathrm{a}}$ & $0.67 \pm 0.06^{\mathrm{a}}$ \\
\hline
\end{tabular}

Data are expressed as the mean \pm standard deviation. ${ }^{\mathrm{a}} \mathrm{P}<0.05$ vs. Blank group. DMSO, dimethyl sulfoxide.

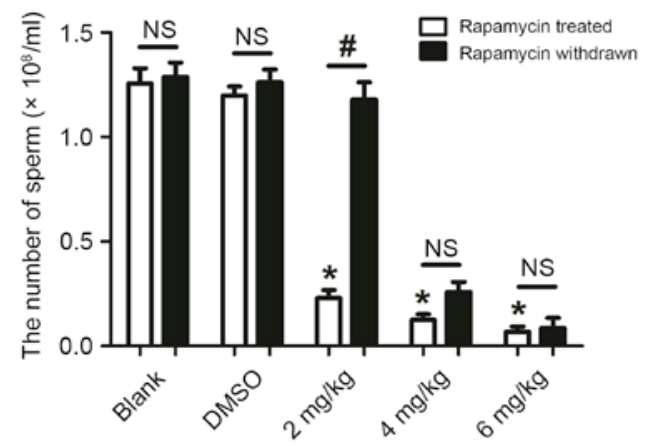

Figure 1. Number of sperm after the treatment and withdrawal of rapamycin. Data are presented as the mean \pm standard deviation (n=10/group). " $\mathrm{P}<0.05$ vs. Blank group; ${ }^{*} \mathrm{P}<0.05$ as indicated. NS, non-significant; DMSO, dimethyl sulfoxide.
A

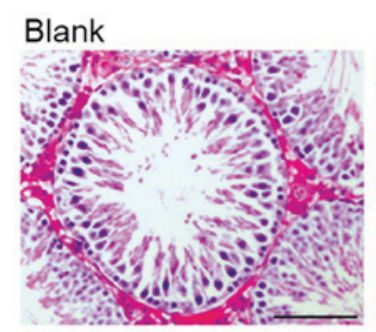

$2 \mathrm{mg} / \mathrm{kg}$

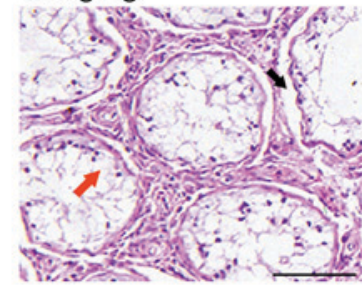

$4 \mathrm{mg} / \mathrm{kg}$

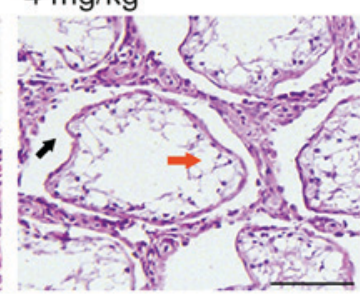

$6 \mathrm{mg} / \mathrm{kg}$

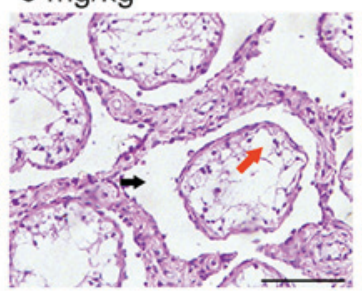

B

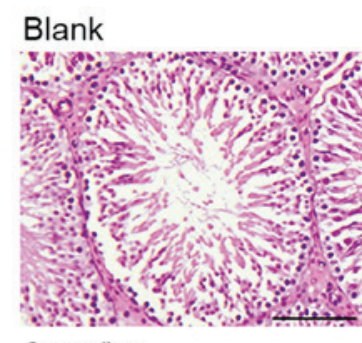

$2 \mathrm{mg} / \mathrm{kg}$

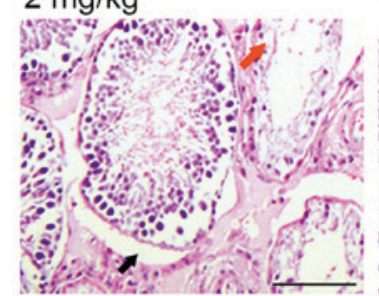

DMSO

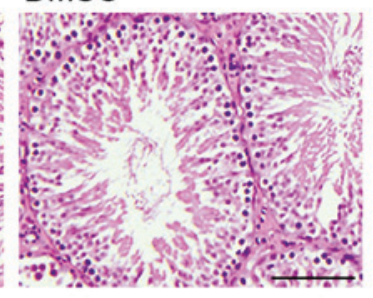

$4 \mathrm{mg} / \mathrm{kg}$

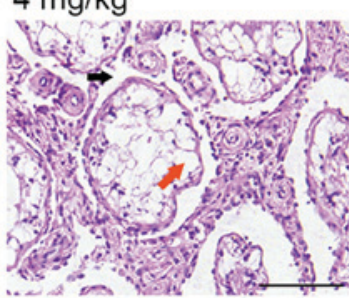

$6 \mathrm{mg} / \mathrm{kg}$

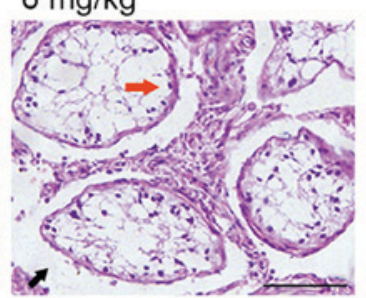

Figure 2. Hematoxylin and eosin staining of the testes after (A) treatment and (B) withdrawal of rapamycin (magnification, $x 400$ ). Red arrows indicate vacuolization of the seminiferous epithelium, and black arrows indicate interstitium of seminiferous tubule. Scale bar=50 $\mu \mathrm{m}$. DMSO, dimethyl sulfoxide.

in control groups, spermatogenic cells (including spermatogonium, primary spermatocytes, secondary spermatocytes and spermatids from outside to inside layer of the seminiferous tubule) were easily visible. However, in the testes of rats in the 2,4 and $6 \mathrm{mg} / \mathrm{kg}$ groups, extensive testicular atrophy with vacuolization of the seminiferous epithelium appeared, and spermatogenic cells were not identifiable (Fig. 2A). Additionally, hyperplasia of testicular interstitial cells (the cells among seminiferous tubules, also named Leydig cells) were observed in rapamycin-treated groups (Fig. 2A). 
A

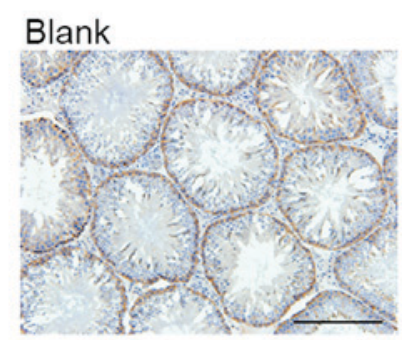

$2 \mathrm{mg} / \mathrm{kg}$

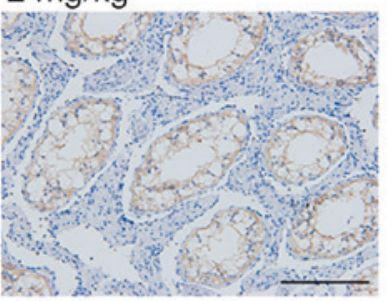

DMSO

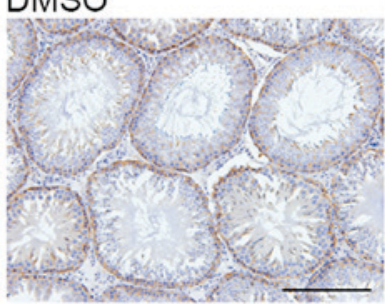

$4 \mathrm{mg} / \mathrm{kg}$

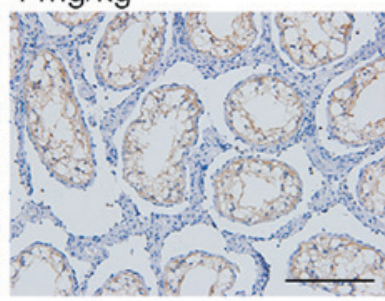

NTC

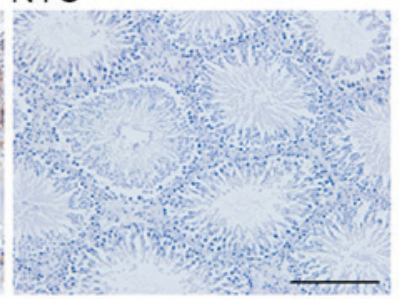

$6 \mathrm{mg} / \mathrm{kg}$

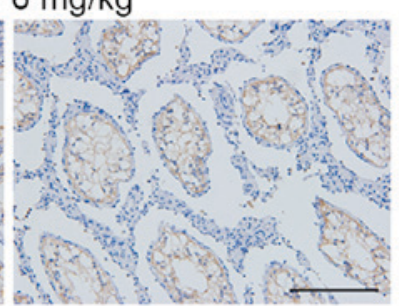

B

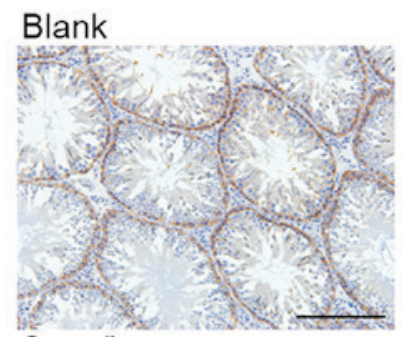

\section{DMSO}

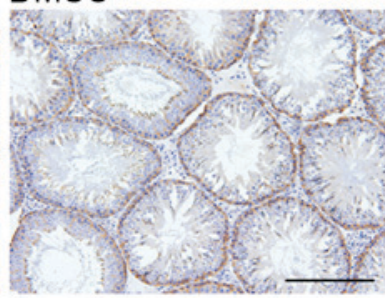

$2 \mathrm{mg} / \mathrm{kg}$

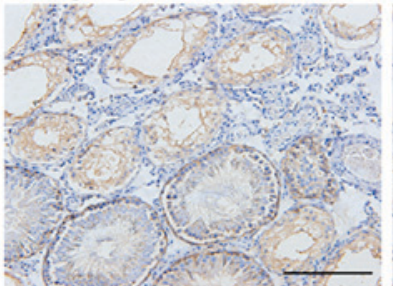

$4 \mathrm{mg} / \mathrm{kg}$

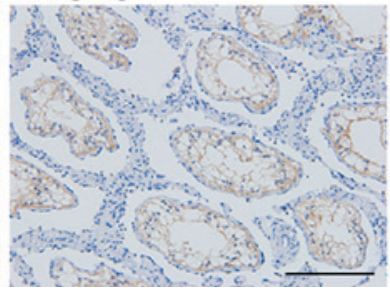

$6 \mathrm{mg} / \mathrm{kg}$

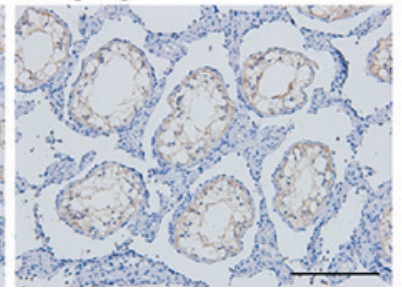

Figure 3. Immunohistochemistry of mTOR in rat testes tissue after (A) treatment and (B) withdrawal of rapamycin. The yellowish-brown staining indicates a positive signal. Magnification, x200; scale bar=100 $\mu \mathrm{m}$. DMSO, dimethyl sulfoxide; NTC, negative control; mTOR, mechanistic target of rapamycin.

In addition, the interstitium between the seminiferous tubule and testicular interstitial cells increased obviously in rapamycin-treated groups, in a dose-dependent manner (Fig. 2A).

Consistent with the results of sperm calculation, morphology of seminiferous tubules in the $2 \mathrm{mg} / \mathrm{kg}$ group were partially recovered except for in the 4 and $6 \mathrm{mg} / \mathrm{kg}$ groups after withdrawal of rapamycin (Fig. 2B). Spermatogenic cells were observed in part of the seminiferous tubules of rats in the $2 \mathrm{mg} / \mathrm{kg}$ rapamycin withdrawal group, and the vacuolization of the seminiferous epithelium decreased. However, the histological structure of testes in the 4 and $6 \mathrm{mg} / \mathrm{kg}$ groups did not present an obvious recovery after withdrawal of rapamycin for 24 weeks.

Changes of mTOR and Ki67 in the testes of rats after treatment and withdrawal of rapamycin. To confirm the inhibition effect of rapamycin on mTOR, immunohistochemistry of mTOR in testes tissue of rats was performed. In the Blank and DMSO groups, mTOR was located at the cytoplasm of cells in the outer layer of the seminiferous tubules, spermatogonia and primary spermatocytes (Fig. 3A). As the structure of seminiferous tubules was damaged in the 2,4 and $6 \mathrm{mg} / \mathrm{kg}$ rapamycin-treated groups, mTOR was not detected in these groups. After withdrawal of rapamycin for 24 weeks, morphology of seminiferous tubules in the $2 \mathrm{mg} / \mathrm{kg}$ group partially recovered to normal levels, and mTOR was detectable again (Fig. 3B).

To explore if the reduction of sperm number was caused by the dysfunction of spermatogenesis, immunohistological staining for the proliferation marker Ki67, a down-stream target of mTOR, was performed. Compared with the Blank and DMSO groups, Ki67 positive cells within seminiferous tubules were markedly decreased in the testes of rapamycin-treated groups (Fig. 4A). Expression of Ki67 was associated with the degree of atrophy in the seminiferous tubules. After withdrawal of rapamycin for 24 weeks, Ki67 was expressed in the recovered seminiferous tubules of rats in the $2 \mathrm{mg} / \mathrm{kg}$ group (Fig. 4B).

Changes of autophagy in testes of rats following treatment and withdrawal of rapamycin. To further explore the molecular mechanism by which rapamycin inhibits spermatogenesis, the expression of autophagy-associated genes in the testes was detected using western blotting (Fig. 5). LC3 II was upregulated in the $2 \mathrm{mg} / \mathrm{kg}$ group, while P62 was downregulated, indicating 
A
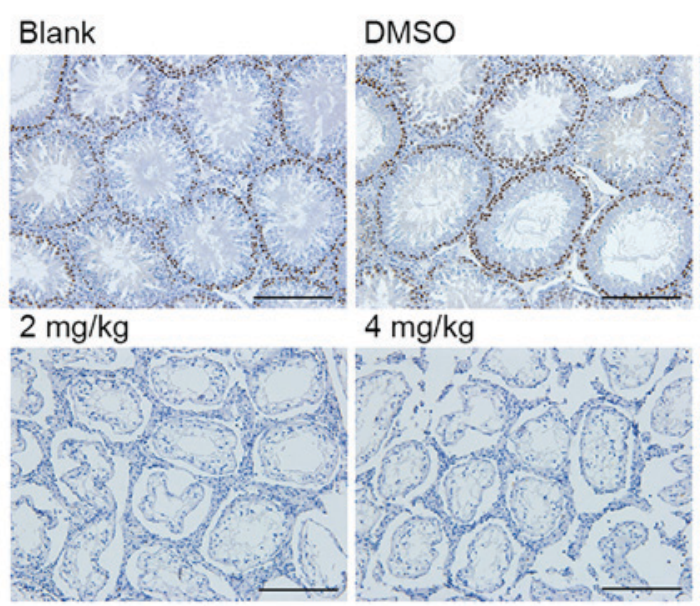

$4 \mathrm{mg} / \mathrm{kg}$

NTC

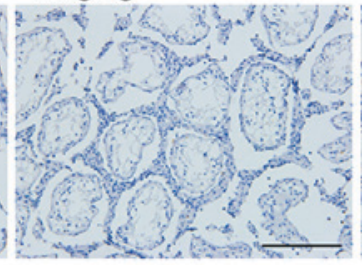

$6 \mathrm{mg} / \mathrm{kg}$
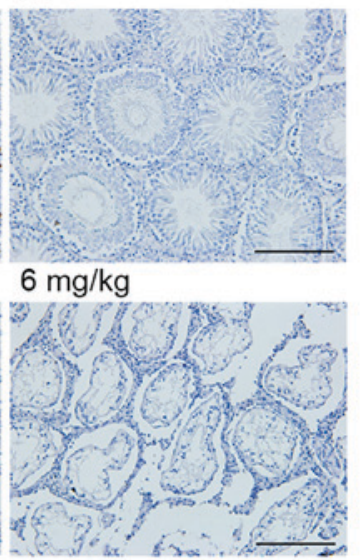

B

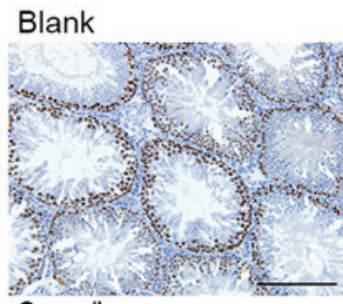

DMSO

$2 \mathrm{mg} / \mathrm{kg}$

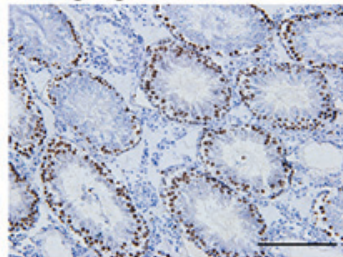

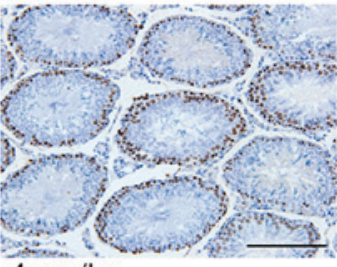

$4 \mathrm{mg} / \mathrm{kg}$

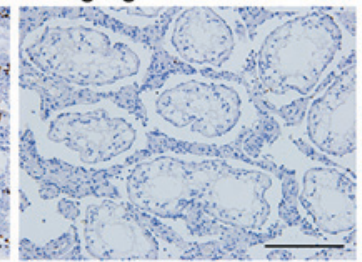

$6 \mathrm{mg} / \mathrm{kg}$

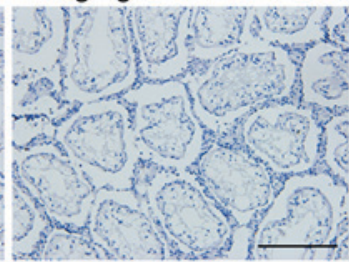

Figure 4. Immunohistochemistry of Ki67 in rat testes tissue after (A) treatment and (B) withdrawal of rapamycin. The yellowish-brown staining indicates a positive signal. Magnification, x200; scale bar=100 $\mu \mathrm{m}$. DMSO, dimethyl sulfoxide; NTC, negative control.

autophagy is activated. Both LC3 II and P62 protein expression levels were reduced in the testes of rats from the 4 and $6 \mathrm{mg} / \mathrm{kg}$ groups, suggesting that there was over-activation of autophagy and these associated proteins were degraded by lysosomes (Fig. 5A and C). The expression of LC3 II and P62 in the $2 \mathrm{mg} / \mathrm{kg}$ withdrawal group was similar with the control group. However, the expression of autophagy-associated proteins decreased in the 4 and $6 \mathrm{mg} / \mathrm{kg}$ withdrawal groups (Fig. 5B and D).

Changes of testis cell apoptosis following treatment and withdrawal of rapamycin. Western blotting was used to detect the expression of apoptosis-associated proteins in the rat testes (Fig. 6). The protein expression level of Bax increased following treatment with rapamycin. The expression of Bcl-2 in the $2 \mathrm{mg} / \mathrm{kg}$ group was similar compared with the control group, whereas it was downregulated in the 4 and $6 \mathrm{mg} / \mathrm{kg}$ groups (Fig. 6A and C). After withdrawal for 24 weeks, there were no differences in the expression levels of Bax and Bcl-2 between the control and $2 \mathrm{mg} / \mathrm{kg}$ groups, whereas Bax protein expression levels increased and Bcl-2 protein expression levels decreased in a dose-dependent manner (Fig. 6B and D).

Changes of $p$-p70S6K/p70S6K levels in testis following treatment and withdrawal of rapamycin. To explore the potential underlying mechanism of the blockage of rapamycin on spermatogenesis, the ratio of p-p70S6K/p70S6K was assessed by western blotting. Total p70S6K protein expression levels were unchanged between the 5 groups with and without treatment of rapamycin; however, p-p70S6K protein expression levels were markedly reduced in rapamycin-treated rats compared with control rats (Fig. 7A). The ratio of p-p70S6K/p70S6K was in accordance with the expression of p-p70S6K (Fig. 7B). The ratio of $\mathrm{p}-\mathrm{p} 70 \mathrm{~S} 6 \mathrm{~K} / \mathrm{p} 70 \mathrm{~S} 6 \mathrm{~K}$ was calculated through this formula: phospho-p70S6K/p70S6K=(phospho-p70S6K/ $\beta$-actin) $/(\mathrm{p} 70 \mathrm{~S} 6 \mathrm{~K} / \beta$-actin). Following withdrawal of the treatment of rapamycin, phosphorylation of p70S6K increased markedly in the $2 \mathrm{mg} / \mathrm{kg}$ group (Fig. 7C and D). However, there was no obvious increase in the 4 and $6 \mathrm{mg} / \mathrm{kg}$ groups after withdrawal of rapamycin for 24 weeks (Fig. 7C and D).

\section{Discussion}

Infertility affects $19 \%$ of the general population; additional attention should be paid to paid to male factors due to the high prevalence of male infertility (19,20). Male subfertility and infertility are mainly caused by genetic factors, environmental factors and medication (21-24). Oligozoospermia is the most common etiological factor responsible for male infertility (24). Several previous case reports have indicated that rapamycin could induce male infertility (24), but the potential mechanisms are unclear. 
A

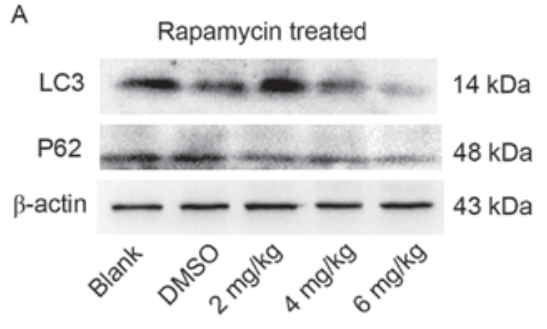

C
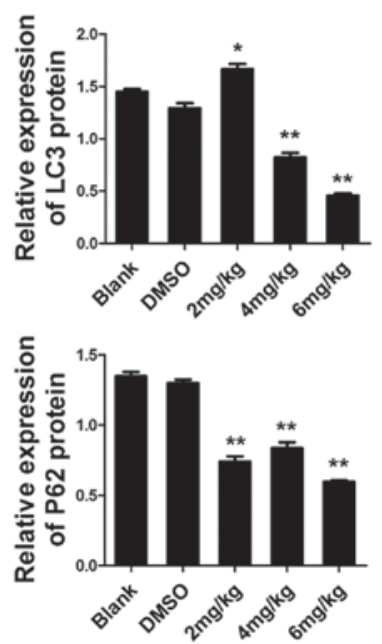

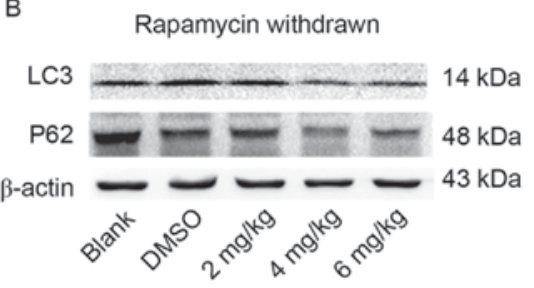

D
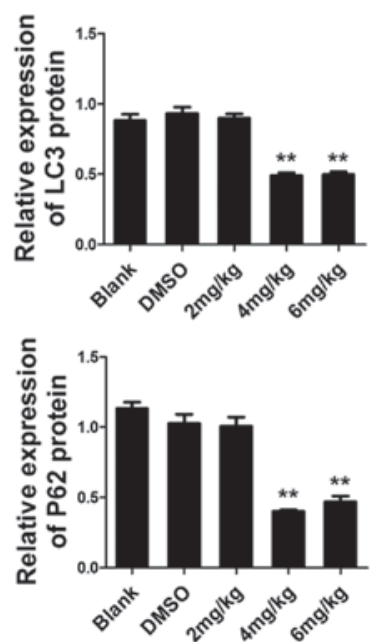

Figure 5. Protein expression levels of LC3 and P62 in the testes of rats after (A) treatment and (B) withdrawal of rapamycin, as detected by western blotting. $\beta$-actin served as a loading control. Statistical analysis of the protein expression of LC3 and P62 in the testes following (C) treatment with rapamycin and (D) withdrawal of rapamycin. ${ }^{*} \mathrm{P}<0.05$ and ${ }^{* *} \mathrm{P}<0.01$ vs. Blank. LC3, microtubule-associated protein 1A/1B-light chain 3; DMSO, dimethyl sulfoxide.

A

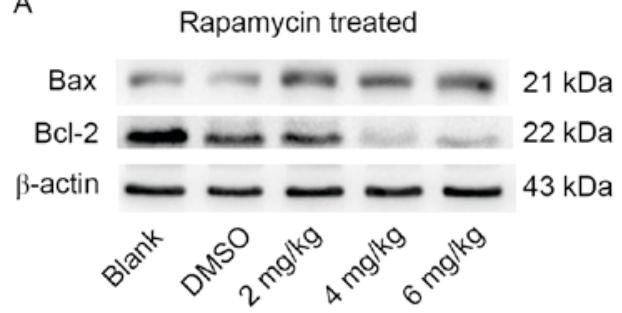

C
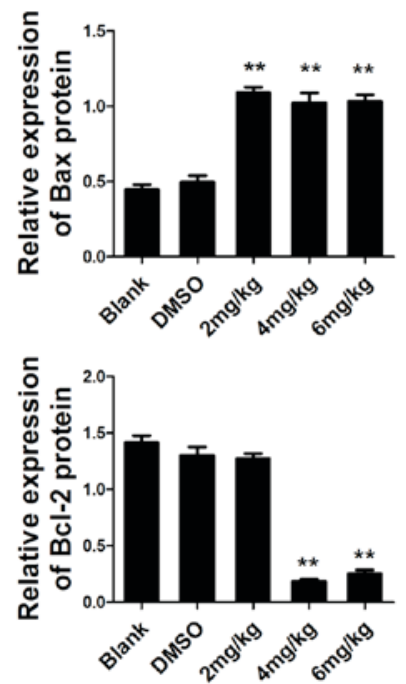

\section{Rapamycin withdrawn}

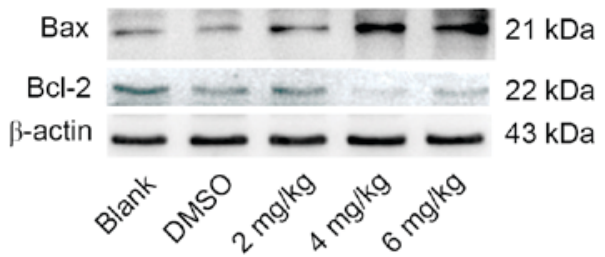

D
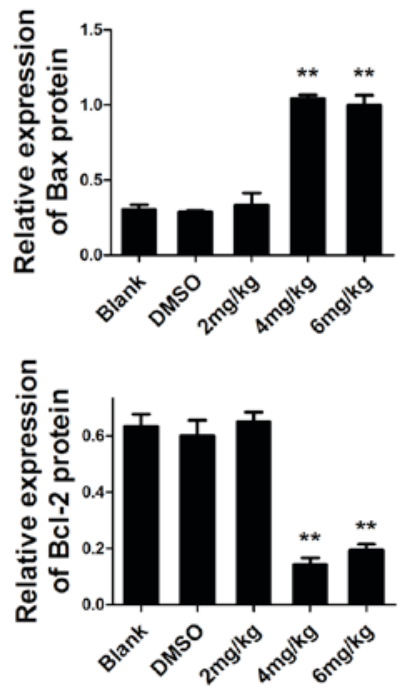

Figure 6. Protein expression levels of Bax and Bcl-2 in the testes of rats after (A) treatment and (B) withdrawal of rapamycin, as detected by western blotting. $\beta$-actin served as a loading control. Statistical analysis of the protein expression of Bax and Bcl-2 in the testes following (C) treatment with rapamycin and (D) withdrawal of rapamycin. ${ }^{* *} \mathrm{P}<0.01$ vs. Blank. DMSO, dimethyl sulfoxide; Bcl-2, B-cell lymphoma 2; Bax, Bcl-2 X associated protein. 
A

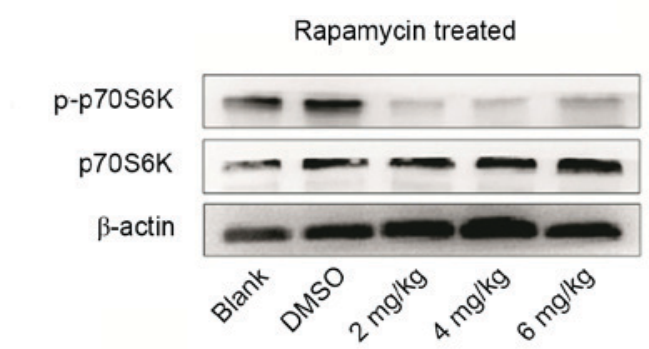

C

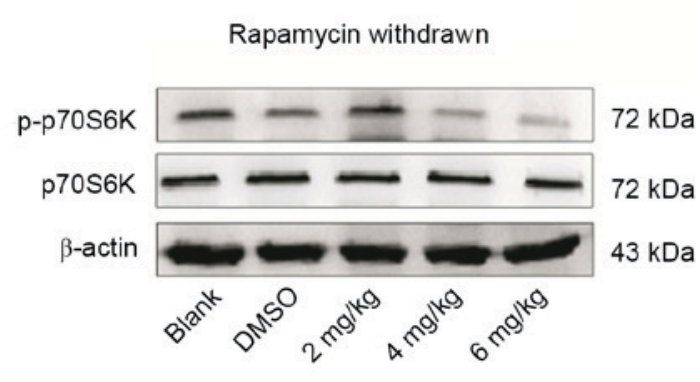

B

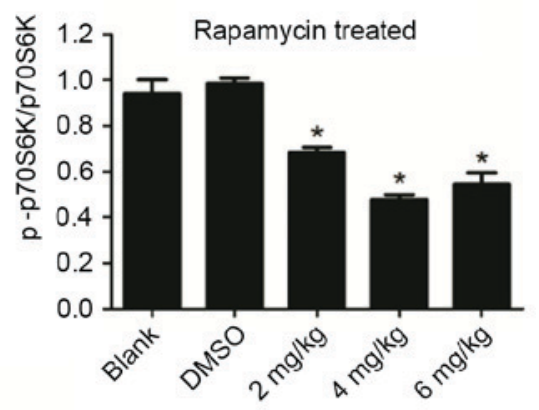

D

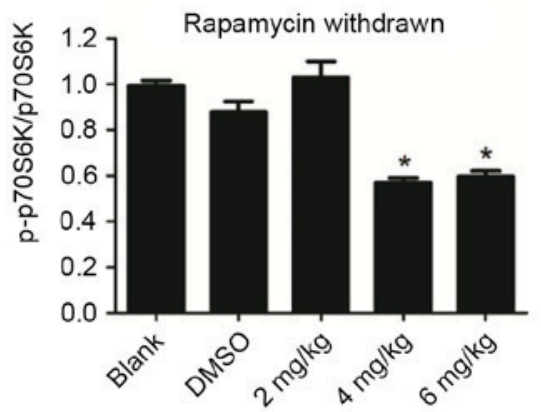

Figure 7. Protein expression levels of p-p70S6K and p70S6K in the testes of rats after treatment and withdrawal of rapamycin, as detected by western blotting. $\beta$-actin served as a loading control. (A) Representative western blot images and (B) quantification after rapamycin treatment. (C) Representative western blot images and (D) quantification after rapamycin withdrawal. Data are presented as the mean \pm standard deviation. *P<0.05 vs. Blank. DMSO, dimethyl sulfoxide; p, phosphorylated; p70S6K, p70S6 kinase.

The present study demonstrated that rapamycin treatment markedly reduced the number of sperm in rats, consistent with a previous report (5). To explore the potential mechanisms by which the sperm number decreased in rapamycin-treated groups, body and testes weights of the rats were measured and organ coefficients were calculated. The results demonstrated that the organ coefficients of testes in all the rapamycin-treated groups were reduced compared with the control groups. HE staining results demonstrated that diameters of spermatogonial nuclei in the rapamycin-treated groups were much shorter than the control groups. Furthermore, seminiferous tubules appeared to have extensive testicular atrophy with vacuolization of the seminiferous epithelium in rapamycin-treated groups, which was consistent with the reduction of the weight of the testes. These findings indicated that the reduced sperm number in rapamycin-treated rats may because of spermatogenesis blockage.

As rapamycin is a specific inhibitor of mTOR (11), immunohistochemistry of mTOR in testes of rats after rapamycin treatment was performed, and the results indicated that mTOR was suppressed by rapamycin. Furthermore, immunostaining of Ki67 demonstrated that the spermatogenesis process was blocked. These findings suggested that the reduced sperm number in rapamycin-treated rats was due to the blockage of spermatogonia proliferation. Autophagy is an evolutionary conserved process by formation of the autophagosome, thus updating cellular metabolites and organelles. mTOR is a key regulator of the autophagy signaling pathway (25). Rapamycin could activate autophagy of cells by inhibiting mTOR (26). The present study revealed that low doses of rapamycin could lead to activation of autophagy in rat testes. This may be a self-protection mechanism of the cell in response to external stress. Thus, spermatogenesis can be recovered in the testes from rats in the low dose group. This was consistent with a previous report, in which rapamycin was used to reduce podocyte damage by activating autophagy through inhibition of the mTOR-ULK1 channel (27). High doses of rapamycin may result in over-activation of autophagy, eventually leading to excessive consumption of autophagic proteins; the damage from which cannot be compensated. In addition, the present study demonstrated that cell apoptosis increased after treatment with rapamycin. More work is needed to clarify the specific molecular mechanism by which rapamycin induces apoptosis, and the interaction between autophagy and apoptosis in the testes.

P70S6K is a typically recognized downstream target of mTOR, whose phosphorylation is critical for proliferation. p-p70S6K can facilitate the translation of mRNA and promote cell proliferation $(28,29)$. mTOR elicits its functions mainly through controlling protein synthesis by phosphorylating and activating the ribosomal protein S6 kinase (S6K) (30). In the present study, p-p70S6K and the ratio of pp70S6K/p70S6K was much lower in rapamycin-treated groups compared with control groups, which was consistent with inhibition of rapamycin on $\mathrm{mTOR}$. Taken together, these results indicated that rapamycin inhibited the proliferation of spermatogonia via inhibiting phosphorylation of p70S6K, and thereby resulted in the reduction of sperm number and impairment of the seminiferous tubule. The degree of the impairment was dose-dependent.

Notably, it was demonstrated that the damaged structure of seminiferous tubules and reduced number of sperm was reversible after withdrawal of rapamycin, which is consistent with previous research (3). However, this reversible phenomenon 
appeared only in rats of $2 \mathrm{mg} / \mathrm{kg} /$ day group after withdrawal of rapamycin for 24 weeks. The rats in 4 and $6 \mathrm{mg} / \mathrm{kg} /$ day groups did exhibit significant recovery. Therefore, rapamycin may have toxicological effects on the testes, and the damage is reversible only at a certain low dose. Over the past few decades, the prevalence of people with a renal transplant has been increasing in Europe (31). Although rapamycin is a powerful immunosuppressant drug, the depression of spermatogenesis is a significant side effect at least in younger men. Approximately 30-40\% of men receiving kidney transplantation are younger than 50 , and fertility is of major concern (32). Therefore, the dose of rapamycin has to be taken into consideration, particularly when administered to male patients who intend to have children. The patients should be informed of this side effect, and the dose of rapamycin should be controlled to an appropriate range.

In conclusion, the present study demonstrated that rapamycin inhibits the proliferation of spermatogonia and damages the structure of the seminiferous tubule through suppressing mTOR signaling pathway and changing the autophagy status, thus resulted in reduction of sperm number. The cessation of rapamycin treatment at a low dose can induce a partial recovery of spermatogenesis. These findings provide important guidance for the clinical application of rapamycin.

\section{Acknowledgements}

The present study was supported by the National Natural Science Foundation of China (grant no. 31501207). The authors would like to thank all the members in our research group for their technical support and Yongjiang Zhou for writing assistance.

\section{References}

1. Abraham RT and Wiederrecht GJ: Immunopharmacology of rapamycin. Annu Rev Immunol 14: 483-510, 1996.

2. Faivre S, Kroemer G and Raymond E: Current development of mTOR inhibitors as anticancer agents. Nat Rev Drug Discov 5: 671-688, 2006

3. Boobes Y, Bernieh B, Saadi H, Raafat Al Hakim M and Abouchacra S: Gonadal dysfunction and infertility in kidney transplant patients receiving sirolimus. Int Urol Nephrol 42: 493-498, 2010

4. Rovira J, Diekmann F, Ramirez-Bajo MJ, Bañón-Maneus E, Moya-Rull D and Campistol JM: Sirolimus-associated testicular toxicity: Detrimental but reversible. Transplantation 93: 874-879, 2012.

5. Deutsch MA, Kaczmarek I, Huber S, Schmauss D, Beiras-Fernandez A, Schmoeckel M, Ochsenkuehn R, Meiser B, Mueller-Hoecker J and Reichart B: Sirolimus-associated infertility: Case report and literature review of possible mechanisms. Am J Transplant 7: 2414-2421, 2007.

6. Singh K and Jaiswal D: Human male infertility: A complex multifactorial phenotype. Reprod Sci 18: 418-425, 2011.

7. Yoshinaga K, Nishikawa S, Ogawa M, Hayashi S, Kunisada T, Fujimoto $\mathrm{T}$ and Nishikawa $\mathrm{S}$ : Role of c-kit in mouse spermatogenesis: identification of spermatogonia as a specific site of c-kit expression and function. Development 113: 689-699, 1991.

8. Vogt PH: Genetics of idiopathic male infertility: Y chromosomal azoospermia factors (AZFa, AZFb, AZFc). Baillieres Clin Obstet Gynaecol 11: 773-795, 1997.

9. Smith RW, Anderson RC, Smith JW, Brook M, Richardson WA and Gray NK: DAZAP1, an RNA-binding protein required for development and spermatogenesis, can regulate mRNA translation. RNA 17: 1282-1295, 2011.

10. Wullschleger S, Loewith $\mathrm{R}$ and Hall MN: TOR signaling in growth and metabolism. Cell 124: 471-484, 2006.
11. Singha UK, Jiang Y, Yu S, Luo M, Lu Y, Zhang J and Xiao G: Rapamycin inhibits osteoblast proliferation and differentiation in MC3T3-E1 cells and primary mouse bone marrow stromal cells. J Cell Biochem 103: 434-446, 2008.

12. Buckmaster PS and Lew FH: Rapamycin suppresses mossy fiber sprouting but not seizure frequency in a mouse model of temporal lobe epilepsy. J Neurosci 31: 2337-2347, 2011.

13. Zeng LH, Rensing NR and Wong M: The mammalian target of rapamycin signaling pathway mediates epileptogenesis in a model of temporal lobe epilepsy. J Neurosci 29: 6964-6972, 2009.

14. Liu G, Han F, Yang Y, Xie Y, Jiang H, Mao Y, Wang H, Wang M, Chen R, Yang J and Chen J: Evaluation of sphingolipid metabolism in renal cortex of rats with streptozotocin-induced diabetes and the effects of rapamycin. Nephrol Dial Transplant 26: 1493-1502, 2011.

15. Xu H, Shen L, Chen X, Ding Y, He J, Zhu J, Wang Y and Liu X: mTOR/P70S6K promotes spermatogonia proliferation and spermatogenesis in Sprague Dawley rats. Reprod Biomed Online 32: 207-217, 2016.

16. Zhou Y, Zhang H, He J, Chen X, Ding Y, Wang Y and Liu X: Effects of sodium fluoride on reproductive function in female rats. Food Chem Toxicol 56: 297-303, 2013.

17. World Health Organization: Laboratory manual of the WHO for the examination of human semen and sperm-cervical mucus interaction. Ann Ist Super Sanita 37: I-XII, 1-123, 2001 (In Italian).

18. Liu S, He J, Chen X, Ding Y, Geng Y, Wu M, Liu X and Wang Y: Costimulatory molecule CD28 participates in the process of embryo implantation in mice. Reprod Sci 21: 686-695, 2014.

19. Ugwuja EI, Ugwu NC and Ejikeme BN: Prevalence of low sperm count and abnormal semen parameters in male partners of women consulting at infertility clinic in Abakaliki, Nigeria. Afr J Reprod Health 12: 67-73, 2008.

20. Ikechebelu JI, Adinma JI, Orie EF and Ikegwuonu SO: High prevalence of male infertility in southeastern Nigeria. J Obstet Gynaecol 23: 657-659, 2003.

21. Dada R, Gupta NP and Kucheria K: Cytogenetic and molecular analysis of male infertility: Y chromosome deletion during nonobstructive azoospermia and severe oligozoospermia. Cell Biochem Biophys 44: 171-177, 2006.

22. Multigner L and Oliva A: Environment as a risk factor for male infertility. ScientificWorldJournal 1: 576-578, 2001.

23. Sharpe RM: Environment, lifestyle and male infertility. Baillieres Best Pract Res Clin Endocrinol Metab 14: 489-503, 2000

24. Skrzypek J and Krause W: Azoospermia in a renal transplant recipient during sirolimus (rapamycin) treatment. Andrologia 39: 198-199, 2007.

25. Petiot A, Ogier-Denis E, Blommaart EF, Meijer AJ and Codogno P: Distinct classes of phosphatidylinositol 3'-kinases are involved in signaling pathways that control macroautophagy in HT-29 cells. J Biol Chem 275: 992-998, 2000.

26. Santos RX, Cardoso S, Correia S, Carvalho C, Santos MS and Moreira PI: Targeting autophagy in the brain: A promising approach? Cent Nerv Syst Agents Med Chem 10: 158-168, 2010.

27. Wu L, Feng Z, Cui S, Hou K, Tang L, Zhou J, Cai G, Xie Y, Hong Q, Fu B and Chen X: Rapamycin upregulates autophagy by inhibiting the mTOR-ULK1 pathway, resulting in reduced podocyte injury. PLoS One 8: e63799, 2013.

28. Pene F, Claessens YE, Muller O, Viguié F, Mayeux P, Dreyfus F, Lacombe $C$ and Bouscary D: Role of the phosphatidylinositol 3-kinase/Akt and mTOR/P70S6-kinase pathways in the proliferation and apoptosis in multiple myeloma. Oncogene 21: 6587-6597, 2002

29. Fingar DC, Richardson CJ, Tee AR, Cheatham L, Tsou C and Blenis J: mTOR controls cell cycle progression through its cell growth effectors S6K1 and 4E-BP1/eukaryotic translation initiation factor 4E. Mol Cell Biol 24: 200-216, 2004.

30. Montagne J, Stewart MJ, Stocker H, Hafen E, Kozma SC and Thomas G: Drosophila S6 kinase: A regulator of cell size. Science 285: 2126-2129, 1999.

31. Stengel B, Billon S, Van Dijk PC, Jager KJ, Dekker FW, Simpson K and Briggs JD: Trends in the incidence of renal replacement therapy for end-stage renal disease in Europe, 1990-1999. Nephrol Dial Transplant 18: 1824-1833, 2003.

32. Huyghe E, Zairi A, Nohra J, Kamar N, Plante P and Rostaing L: Gonadal impact of target of rapamycin inhibitors (sirolimus and everolimus) in male patients: An overview. Transpl Int 20: 305-311, 2007. 